Paolo Formaggioni, ${ }^{\star}$ Massimo Malacarne, Piero Franceschi, Andrea Summer

\title{
Influenza del management e dell'alimentazione animale sulla resa in formaggio Parmigiano Reggiano e Grana Padano: una minireview
}

\section{Influence of management and animal feeding on cheese yield in Parmigiano Reggiano and Grana Padano: a minireview}

Dipartimento di Scienze Medico-Veterinarie, Università degli Studi di Parma, Via del Taglio 10, 43126, Parma, Italy

\section{*Corresponding author:}

Paolo Formaggioni

Dipartimento di Scienze Medico-Veterinarie, Università degli Studi di Parma, Via del Taglio

10, I-43126, Parma. Tel. 0521-032614 Email: paolo.formaggioni@unipr.it
Ricevuto il 19 novembre 2019

Accettato il 3 febbraio 2020

DOI: $10.36138 / S T L C .01 .2020 .02$

\section{Riassunto}

La rassegna si propone di valutare l'influenza del management e dell'alimentazione animale sulla resa in formaggio Parmigiano Reggiano e Grana Padano, recensendo tutti i lavori che in letteratura si sono occupati di questa tematica. In particolare, per quanto riguarda la resa in formaggio Parmigiano Reggiano, è stata valutata la sostituzione della soia con fava e pisello nella dieta delle bovine, le differenze tra latte prodotto in allevamenti a stabulazione fissa e latte prodotto a allevamenti a stabulazione libera, gli ef- fetti della somministrazione alle bovine di monensina a rilascio programmato per prevenire la chetosi per quanto riguarda la resa in formaggio Grana Padano è stata valutata la sostituzione parziale o totale di insilato di orzo all'insilato di mais nell'alimentazione delle bovine.
Parole chiave:
resa in formaggio
- Parmigiano Reggiano
- Grana Padano
- Management
- Alimentazione animale

\begin{abstract}
The review aims to investigate the influence of management and animal feeding on Parmigiano Reggiano and Grana Padano cheese yield, reviewing all the studies that in the literature have dealt with this issue. In particular, the following aspects are taken into consideration: concerning the Parmigiano Reggiano cheese yield, the substitution of soya with fava beans and peas in the cows' diet, the differences between milk produced in tie stall barn and milk produced in free stall barn, the effects of administration of monensin with scheduled release to
\end{abstract}

prevent ketosis in cattle; concerning the Grana Padano cheese yield, the partial or total replacement of maize silage with barley silage in the feeding of cows.

\section{Keywords:}

Cheese yield

- Parmigiano Reggiano

- Grana Padano

- Management

Animal feeding 


\section{INTRODUZIONE}

Si definisce resa casearia la quantità di formaggio espressa in chilogrammi che si ricava da $100 \mathrm{~kg}$ di latte. Si tratta di un parametro di rilevante importanza: maggiore è la percentuale di solidi recuperata, maggiore è la quantità di formaggio ottenuta e quindi il guadagno in termini economici. Nel processo caseario, pertanto, risulta fondamentale il massimo recupero possibile di solidi del latte nella cagliata.

Si comprende perciò come sia importante valutare quali siano gli effetti che influiscono sul valore della resa casearia, in modo tale da ottimizzarne i parametri.

Nonostante l'importanza, sia economica sia in termini di produzione, di questi due formaggi, in realtà, come potremo riscontrare, i lavori che si sono occupati dell'influenza del management (fattori di tipo zootecnico) e dell'alimentazione delle bovine sulla resa in Parmigiano Reggiano e Grana Padano sono pochi, anche se molto distribuiti nel corso degli anni. I quattro contributi che vengono di seguito descritti, sono, a nostra conoscenza, gli unici presenti in letteratura.

\section{SOSTITUZIONE DELLA SOIA CON FAVA E PISELLO NELLA DIETA DELLE BOVINE: EFFETTI SULLA RESA IN PARMIGIANO REGGIANO}

Nello studio di Mordenti et al. [1], due gruppi omogenei di vacche da latte (per un totale di 119 vacche), dopo un periodo di adattamento di 2 settimane, sono stati alimentati con la stessa dieta $\mathrm{d}$ base aggiunta con un concentrato contenente il $10 \%$ di farina di soia (Primo gruppo) o il $10,1 \%$ di fava e il $10,1 \%$ di piselli (Secondo gruppo). La sostituzione della farina di soia con fava + pisello ha ridotto l'assunzione di sostanza secca (-0,83 kg/capo/giorno, equivalente al $3,5 \%)$ e la produzione di latte $(-1,29 \mathrm{~kg} /$ capo/giorno, equivalente al 3,9\%).
La qualità del latte (grasso, caseina, lattosio, urea, cellule somatiche, acidità titolabile e tempo di coagulazione) non è stata influenzata dalla dieta. La dieta fava + piselli ha aumentato significativamente i contenuti di grasso $(P<0,01)$ e caseina $(P<0,05)$ del latte in caldaia. L'analisi delle proprietà di coagulazione del latte non ha mostrato differenze significative tra i trattamenti. La quantità di formaggio (a 32 ore) prodotta da $100 \mathrm{~kg}$ di latte in caldaia era significativamente maggiore nel gruppo fava + piselli, probabilmente a causa del maggiore contenuto di grasso e caseina del latte in caldaia; dopo 1 anno di stagionatura questa tendenza era ancora osservabile ma le differenze non erano più significative. Nella Tabella I sono riportati, in dettaglio, i risultati ottenuti da Mordenti et al. [1] per la resa a 32 ore e dopo 1 anno di latti di bovine alimentate con farina di soia e latte di bovine alimentate con fava + pisello.

I risultati di questa ricerca hanno mostrato che è possibile sostituire la farina di soia con fave e piselli nella dieta delle vacche da latte; la riduzione dell'assunzione di mangime e della produzione di latte è parzialmente compensata dall'aumento del contenuto di grasso e caseina del latte e della resa in formaggio a 32 ore.

\section{LATTE PRODOTTO IN ALLEVAMENTI IN STABULAZIONE FISSA E LATTE PRODOTTO IN ALLEVAMENTI A STABULAZIONE LIBERA NELLA CASEIFICAZIONE A PARMIGIANO REGGIANO}

Lo scopo dello studio di Summer et al. [2] è stato quello di confrontare le caratteristiche del latte di massa prodotto in allevamenti a stabulazione fissa con quello prodotto in allevamenti a stabulazione libera, nella zona di produzione del formaggio Parmigiano Reggiano. Quattordici allevamenti a stabulazione fissa e 14 allevamenti a stabulazione libera sono stati coinvolti nello studio. I campioni di latte di massa sono stati raccolti mensilmente da ciascun allevamento per un periodo di 3 anni. II latte prodotto da vacche in stabu-

\section{Tabella I. Resa a 32 ore e dopo 1 anno di latte di bovine alimentate con farina di soia e latte di bovine alimentate con fava + pisello (Mordenti et al., 2007).}

Table I. Cheese yield at 32 hours and after 1 year of milk from cows fed soya and of milk from cows fed fava beans + peas (Mordenti et al., 2007).

\begin{tabular}{cccc} 
& farina di soia & fava + pisello \\
\hline Resa a 32 ore & $8,44 \%$ & $8,61 \%$ & $* *$ \\
\hline Resa a 1 anno & $7,49 \%$ & $7,62 \%$ & NS \\
\hline
\end{tabular}

${ }^{* *} \mathrm{P}<0,01 ; \mathrm{NS}: \mathrm{P}>0,05$ 
lazione libera ha mostrato valori più elevati di grasso e proteina e cellule somatiche più basse (3,82 vs $3,61 \mathrm{~g} / 100 \mathrm{~g} ; 3,45$ vs 3,30 $\mathrm{g} / 100 \mathrm{~g} ; 158,891$ vs 426,088 cellule/mL, rispettivamente) rispetto al latte raccolto da allevamenti a stabulazione fissa (latte $\mathrm{T}$ ).

Applicando la formula di previsione della resa di Formaggioni et al. [3],

$24 \mathrm{~h} \mathrm{CY}=1,225$ * Grasso $(\mathrm{g} / 100 \mathrm{~g})+1,373$ * Proteina $(\mathrm{g} / 100 \mathrm{~g})+0,445$

le due rese attese risultano essere: 8,86 vs $8,45 \mathrm{~kg} / 100 \mathrm{~kg}$ (libera vs fissa). Bisogna tenere conto che la formula in oggetto è da applicarsi al latte in caldaia; nel lavoro di Summer et al. [2], sono invece presenti i dati del latte intero. Quindi la resa è stata calcolata assumendo le seguenti ipotesi: la proteina del latte in caldaia è stata assunta pari a quella del latte intero. II grasso del latte in caldaia è stato stimato in questo modo: il grasso del latte magro (latte parzialmente scremato per affioramento naturale dal latte della sera), conformemente a quanto riportato da Malacarne et al. [4], è stato stimato circa il 57,18\% di quello del latte intero; il latte in caldaia si è inteso costituito al $50 \%$ da latte magro e da latte intero del mattino.

Nello studio di Summer et al. [2], la percentuale di campioni con profili ottimali di coagulazione risultava più alta per i latti di bovine in stabulazione libera rispetto a quelle in stabulazione fissa. Tuttavia, i latti delle bovine in stabulazione libera presentavano un quantitativo più elevato di spore di clostridi butirrici.

Il latte prodotto dalle bovine allevate nelle stalle a stabulazione libera si è dimostrato più adatto alla produzione del Parmigiano Reggiano sia in termini di resa che di qualità, sebbene si debba considerare la presenza di spore di clostridi, poiché questi microrganismi sono responsabili della maggior parte dei difetti del formaggio Parmigiano Reggiano.

La grande proporzione di campioni con più di 100 spore di clostridi/L nel latte delle bovine allevate nelle stalle a stabulazione libera potrebbe dipendere dalle differenze nelle tecniche di alimentazione tra vacche in stabulazione libera e fissa [5]. Nella maggior parte degli allevamenti in stabulazione libera, i foraggi e i concentrati vengono somministrati alle bovine come unifeed (piatto unico). Poiché non è consentito l'uso dell'insilato, viene aggiunta acqua per legare il concentrato (come farina) al fieno, nella preparazione dell'unifeed. Tuttavia, in questo processo il terreno presente sui foraggi si impasta con il fieno, e viene quindi ingerito dalle bovine [6]. Di conseguenza, è ipotizzabile che le vacche alimentate con il metodo unifeed ingeriranno un numero maggiore di spore di clostridi (che sono principalmente presenti nel suolo) rispetto alle vacche alimentate tradizionalmente, portando a una maggiore escrezione di spore nelle feci. Le feci sono il principale veicolo di trasmissione delle spore di clostridi al latte.

\section{Tabella II. Resa a $\mathbf{3 6}$ ore e a $\mathbf{1 8}$ mesi di latte di bovine trattate con monensina a rilascio programmato (TRT) e latte di bovine del gruppo di controllo (CTR) (Mammi et al., 2018).}

Table II. Cheese yield at 36 hours and at 18 months of milk from cows treated with programmed release monensin (TRT) and milk from control group cows (CTR) (Mammi et al., 2018).

\begin{tabular}{cccc} 
& TRT & CTR & \\
\hline Resa a 36 ore & $8,6 \%$ & $8,9 \%$ & $*$ \\
\hline Resa a 18 mesi & $7,5 \%$ & $7,7 \%$ & $* *$ \\
\hline${ }^{* *} \mathrm{P}<0,01 ;{ }^{*} \mathrm{P}<0,05$ & &
\end{tabular}

\section{EFFETTI SULLA CASEIFICAZIONE A PARMIGIANO REGGIANO DELL'AGGIUNTA DI MONENSINA A RILASCIO PROGRAMMATO}

La chetosi è una delle malattie più importanti negli allevamenti moderni a causa della sua elevata incidenza e dei suoi effetti sulla salute e sulle prestazioni delle bovine. Studi recenti hanno riportato che l'incidenza di chetosi subclinica entro i primi 16 giorni di lattazione varia dal 22 al 43\% sia negli allevamenti europei che negli Stati Uniti.

Mammi et al. [7] hanno studiato gli effetti del trattamento preventivo della chetosi con capsula a rilascio controllato di monensina sul tradizionale processo di trasformazione e sulle caratteristiche finali del formaggio Parmigiano Reggiano. L'uso di questo prodotto di prevenzione per ridurre l'incidenza della chetosi nelle vacche da latte è stato approvato dall'Agenzia europea per i medicinali nel 2013. Nella produzione di formaggio Parmigiano Reggiano, i mangimi, gli additivi per mangimi e i trattamenti per vacche sono rigorosamente regolati per evitare ogni possibile interferenza con i processi di produzione tradizionali. Per questi motivi, in un allevamento in cui tutto il latte veniva utilizzato per la produzione di formaggio Parmigiano Reggiano, la monensina è stata somministrata a 33 vacche, 21 giorni prima del parto, nel gruppo trattato con monensina (TRT), mentre le vacche non trattate, con caratteristiche simili di razza e numero di parti, rappresentavano il gruppo di controllo (CTR). Per 20 settimane, il latte ottenuto da ciascun gruppo e il sieroinnesto corrispondente sono stati gestiti e trasportati separatamente nel caseificio, dove venivano prodotte 2 forme di formaggio per gruppo ogni giorno, per un totale di 552 forme di formaggio Parmigiano Reggiano. 


\section{Tabella III. Resa a 3 mesi, a 6 mesi e a 12 mesi di latte di bovine alimentate con insilato di mais e insilato d'orzo (Migliorati et al., 2017).}

Table III. Cheese yield at 3 months, 6 months and 12 months of milk from cows fed maize silage and barley silage (Migliorati et al., 2017).

\begin{tabular}{cccc}
\hline \multicolumn{1}{l}{ prova } & & & \\
\hline & insilato di mais & insilato di orzo & NS \\
\hline Resa a 3 mesi & $8,16 \%$ & $8,06 \%$ & NS \\
\hline Resa a 6 mesi & $7,84 \%$ & $7,78 \%$ & NS \\
\hline Resa a 12 mesi & $7,73 \%$ & $7,54 \%$ & \\
\hline $\mathbf{2}^{\circ}$ prova & insilato di mais & & NS \\
\hline Resa a 3 mesi & $7,87 \%$ & $8,06 \%$ & NS \\
\hline Resa a 6 mesi & $7,44 \%$ & $7,66 \%$ & NS \\
\hline Resa a 12 mesi & $7,30 \%$ & $7,54 \%$ & orzo \\
\hline
\end{tabular}

NS: differenza non significativa

La composizione del latte di stalla del mattino, le proprietà di caseificazione e le attività di fermentazione del sieroinnesto sono state analizzate due volte a settimana. Ogni aspetto del processo di produzione casearia è stato registrato e il formaggio risultante è stato valutato dopo 36 ore e 6,12 e 18 mesi dalla produzione per resa, difetti di consistenza, composizione e profilo degli acidi grassi. II latte dei 2 gruppi differiva $(P<0,001)$ per il contenuto di cellule somatiche (TRT $=3,40$ vs CTR $=4,06$, punteggio delle cellule somatiche), acidità titolabile $\left(\mathrm{TRT}=3,61\right.$ vs $\left.\mathrm{CTR}=3,69^{\circ} \mathrm{SH} / 50 \mathrm{~mL}\right)$ e contenuto $\mathrm{di}$ caseina (TRT $=2,44$ vs CTR = 2,51\%). I parametri di qualità del sieroinnesto erano comparabili tra i 2 gruppi.

II peso delle forme gemelle, valutato a 36 ore e 18 mesi di stagionatura, era significativamente inferiore $(P<0,01)$ nel gruppo TRT rispetto al gruppo CTR (90,8 contro $93,7 \mathrm{~kg}$ a 36 ore e 79,3 contro $82,0 \mathrm{~kg}$ a 18 mesi). La resa del formaggio (\%) ha mostrato la stessa differenza sia a 36 ore (TRT $=8,6$ rispetto a CTR $=8,9 \%, P<0,05)$ sia dopo 18 mesi di maturazione (TRT $=7,5$ vs CTR $=7,7 \%, P<0,01$ ) (Tabella II). Gli autori affermano che la minore produzione di formaggio del latte trattato potrebbe essere correlata al minor contenuto di caseina del latte, risultato statisticamente significativo (dati riportati sopra).
La composizione finale del formaggio e il profilo organolettico non sono stati influenzati dal trattamento, ad eccezione del contenuto di C18:1, che è risultato aumentato (TRT $=22,8$ vs $C T R=20,8 \%$ sugli acidi grassi totali).

\section{SOSTITUZIONE PARZIALE O TOTALE DI INSILATO DI ORZO ALL'INSILATO DI MAIS NELL'ALIMENTAZIONE DELLE BOVINE: EFFETTI SULLA CASEIFICAZIONE A GRANA PADANO}

Considerando che la disponibilità di acqua per le esigenze agricole è limitata, Migliorati et al. [8] hanno esplorato una possibilità alternativa alla coltivazione del mais nell'alimentazione animale. Questo studio aveva lo scopo di valutare gli effetti di una sostituzione parziale (prova I) o totale (prova II) di mais con l'insilato di orzo nella dieta delle vacche da latte sulla resa e composizione del latte, le sue proprietà di coagulazione, la resa del formaggio e il profilo sensoriale del Grana Padano di 16 mesi.

Non sono stati osservati effetti della dieta, in entrambi le prove, sulla produzione di latte, sulle cellule somatiche, $\mathrm{pH}$ e acidità titolabile. 
Non sono stati osservati effetti anche sul contenuto di proteine e lattosio, né si è registrata alcuna differenza riguardo all'attitudine del latte alla caseificazione, con il tempo di coagulazione (r), la velocità di presa (o tempo di rassodamento del coagulo; $k_{20}$ ) e la consistenza del coagulo $\left(\mathrm{a}_{30}\right)$ che non risultavano influenzati dal trattamento alimentare in nessuna delle due prove. I valori delle rese in formaggio Grana Padano, a diverse età di maturazione, ottenuti da Migliorati et al. [8] per i latti di bovine alimentate con insilato di mais e quelle alimentate con insilato d'orzo sono riportate in Tabella III.

\section{CONCLUSIONI}

In conclusione, si può affermare, sulla scorta dei lavori prima descritti, che è possibile sostituire la farina di soia con fave e piselli nella dieta delle vacche da latte per Parmigiano Reggiano; la resa a 32 ore fa rilevare differenze tra i due trattamenti, risultando significativamente più alta nel gruppo alimentato con fave e piselli; tale differenza, tuttavia, ad 1 anno di stagionatura, non risulta più significativa.

Il latte prodotto dalle bovine allevate nelle stalle a stabulazione libera si è dimostrato più adatto alla produzione del Parmigiano Reggiano sia in termini di resa che di qualità (maggiore contenuto di grasso e proteina, minore contenuto di cellule somatiche, proprietà di coagulazione migliori), sebbene nelle stalle a stabulazione libera si debba considerare una maggiore presenza di spore di clostridi, responsabili della maggior parte dei difetti del formaggio Parmigiano Reggiano.
Nessuna differenza tra i trattamenti alimentari è stata osservata per la resa del formaggio, in entrambe le prove.

Il contenuto di caseina e grasso del latte, nonché il rapporto grasso/caseina, sono considerati i principali fattori che influenzano la resa del formaggio nella produzione di formaggio Grana Padano. Tuttavia, anche se nel primo esperimento è stata osservata una percentuale più alta di grasso nel latte con vacche alimentate con insilato d'orzo, in entrambi gli esperimenti non sono state riscontrate differenze significative nella resa in formaggio.
II latte prodotto da bovine a cui è stata somministrata monensina a rilascio programmato, al fine di prevenzione per ridurre l'incidenza della chetosi, ha fornito una resa in Parmigiano Reggiano significativamente più bassa di circa 0,2-0,3kg/100kg di latte, sia a 36 ore che a 18 mesi di stagionatura. Bisogna comunque tenere conto del fatto che il latte del gruppo trattato partiva già con un contenuto di caseina inferiore.

Per quanto riguarda la resa in formaggio Grana Padano, la sostituzione dell'insilato di mais con l'insilato d'orzo non ha fatto registrare differenze significative sulla produzione e qualità del latte (cellule somatiche, $\mathrm{pH}$ e acidità titolabile, proteine e lattosio, proprietà di coagulazione presamica). In nessuna delle due prove effettuate sono state registrate differenze significative sulla resa in formaggio per i gruppi alimentati con le due diverse diete.

\section{CONFLITTO DI INTERESSE}

Non esistono conflitti di interesse di ordine economico o di altro tipo sull'articolo presentato.

\section{BIBLIOGRAFIA}

1. Mordenti A.L., Merendi F., Fustini M., Formigoni A. Effects of different protein plants in cows diet on milk for Parmigiano Reggiano production. It J Anim Sci 2007; 6(suppl. 1):463-465.

2. Summer A., Franceschi P., Formaggioni P., Malacarne M. Characteristics of raw milk produced by free-stall or tie-stall cattle herds in the Parmigiano-Reggiano cheese production area. Dairy Sci Tech 2014; 94:581-590.

3. Formaggioni P., Summer A., Malacarne M., Franceschi P., Mucchetti G. Italian and Italian-style hard cooked cheeses: predictive formulas for ParmigianoReggiano 24-h cheese yield. Int Dairy J 2015; 51:52-58.

4. Malacarne M., Summer A., Formaggioni P., Franceschi P., Sandri S., Pecorari M., Vecchia P., Mariani P. Dairy maturation of milk used in the manufacture of Parmigiano-Reggiano cheese: effects on physico-chemical characteristics, rennet-coagulation aptitude and rheological properties. J Dairy Res 2008; 75:218-224.
5. Colombari G., Allegretti A., Melani D., Bettoni B., Pecorari M. Growth of clostridial spores in soil, food, faeces, and milk of dairy farm with different levels of technology in production area of Parmigiano-Reggiano. Sci Tecn Latt-Cas 2005; 56:309-344. 6. Pecorari M., Panari G., Guidetti R., Mora R., Summer A. The influence of "unifeed" feeding technique on milk characteristics, cheesemaking technology, quality and typicity of Parmigiano-Reggiano cheese. Sci Tecn Latt-Cas 2001; 52:311-323.

7. Mammi L.M.E., Grazia L., Palmonari A., Canestrari G., Mordenti A., Vecchi M., Formigoni A. Does the dry cow treatment with monensin controlled release capsule affect Parmigiano Reggiano cheese production?. J Dairy Sci 2018; 101:88478859 .

8. Migliorati L., Boselli L., Pirlo G., Moschini M., Masoero F. Corn silage replacement with barley silage in dairy cows' diet does not change milk quality, cheese quality and yield. J Sci Food Agric 2017; 97:3396-3401. 